\title{
Propuesta metodológica para la medición de la satisfacción de usuarios de internet móvil en Colombia*
}

\author{
Methodological Proposal for Measuring the Satisfaction \\ of Mobile Internet Users in Colombia
}

\section{Proposta metodológica para a medição da satisfação de usuários de internet móvel na Colômbia}

\begin{abstract}
Recibido: 6 de mavo de 2019
Revisado: 14 de agosto de 2019

Aceptado: 2 de septiembre de 2019

Harold Aristizábal Uchima*

Universidad Santo Tomas, Colombia

Cómo citar este artículo: Aristizábal Uchima, H. (2020). Propuesta metodológica para la medición de la satisfacción de usuarios de internet móvil en Colombia. Signos. Investigación en Sistemas de Gestión, 12(1), 103-122. DOI: https://doi.org/10.15332/24631140.5423
\end{abstract}

\section{RESUMEN}

En la más reciente actualización al Régimen de Protección de los Derechos de los Usuarios de Servicios de Comunicaciones, se incluyó el requerimiento referente a que los operadores deben monitorear la satisfacción del usuario respecto a cada uno de los medios de atención por medio del indicador denominado Net Promoter Score (NPS). El NPS, a pesar de ser un indicador ampliamente utilizado en diversos sectores, en

** Magíster en Calidad y Gestión Integral. Especialista en Estadística Aplicada. Ingeniero Industrial. Bogotá D. C., Colombia. Correo electrónico: haroldaristizabal@usantotomas.edu.co. Orcid: https://orcid.org/0000-0003-0707-8911 
este caso solo indaga acerca de la satisfacción del usuario frente a la atención recibida por un determinado canal y no permite tener una mirada general de la satisfacción del usuario que abarque todos los aspectos y momentos de la prestación del servicio. En el desarrollo de la investigación, se propone un modelo de medición de la satisfacción de los usuarios de internet móvil, que además de contener el NPS permita medir de una forma representativa y comparable la satisfacción de los usuarios del servicio de internet móvil en las principales ciudades de Colombia, abarcando la experiencia del usuario en todas de las fases del ciclo de prestación del servicio.

Palabras clave: internet móvil, operadores móviles, satisfacción del cliente, medición de la satisfacción, net promoter score.

\section{ABSTRACT}

In the most recent update to the Regime for the Protection of Users' Rights of Communications Services, the requirement was included that operators should monitor user satisfaction with respect to each of the means of attention through the indicator called Net Promoter Score (NPS). The NPS, despite being a widely used indicator in various sectors, in this case it only inquires about user satisfaction against the attention received by a certain channel and it does not allow to have a general view of user satisfaction that covers all aspects and moments of service provision. In the investigation development, a model for measuring the satisfaction of mobile internet users is proposed, which in addition to containing the NPS, allows to measure the satisfaction users of the mobile internet service in a representative and comparable way in the main cities of Colombia, covering the user experience in all phases of the service provision cycle.

Keywords: Mobile Internet, mobile operators, customer satisfaction, satisfaction measurement, net promoter score.

\section{RESUMO}

$\mathrm{Na}$ atualização mais recente do Regime de proteção dos direitos dos usuários de serviços de comunicações, foi incluído o requisito para os operadores monitorarem a satisfação do usuário em relação a cada um dos canais de atendimento através do indicador denominado Net Promoter Score (NPS). O NPS apesar de ser um indicador amplamente utilizado em diferentes setores, neste caso apenas pesquisa sobre a satisfação do usuário em relação à atenção recebida por um determinado canal e não permite ter uma visão geral da satisfação do usuário que envolva todos os aspectos e momentos da prestação do serviço. No desenvolvimento da pesquisa propõe-se um modelo de medição da satisfação dos usuários de internet móvel, que além de conter o NPS, possibilite a medição de uma forma representativa e comparável com a satisfação dos usuários do serviço de internet móvel nas principais cidades da Colômbia, incluindo a experiência do usuário em todas das fases do ciclo de prestação do serviço.

Palavras-chave: Internet móvel, operadores móveis, satisfação do cliente, medição da satisfação, net promoter score. 


\section{INTRODUCCIÓN}

Según el boletín de estimaciones mundiales y regionales de TIC para 2018 publicado por la Unión Internacional de Telecomunicaciones - UIT (2018), se estima que la penetración del internet móvil pasó del 4\% (268 millones de abonados) en 2007 al 69,3\% (5300 millones de abonados) para el cierre de 2018. Los países en desarrollo son los que reportan el mayor crecimiento en la penetración del internet móvil alcanzando un 61\%. Colombia no es ajeno a este panorama mundial, según el reporte de industria del sector TIC publicado por el Ministerio de Tecnologías de la Información y las Comunicaciones MinTIC (2018), para segundo trimestre de 2018 el internet móvil, servicio anexo a la telefonía móvil, presentó una penetración del 52,4\% superando los 25,5 millones de suscriptores durante el segundo trimestre de 2018, lo que significa una variación del $+7,5 \%$ respecto al mismo periodo del año anterior.

En cuanto a la participación de mercado de los operadores que ofrecen el servicio de internet móvil, el $92 \%$ se encuentra concentrado en los tres operadores más grandes del país: Claro (56,9\%), Movistar (22,4\%) y Tigo (12,7\%) (CRC, 2018). Esta situación de concentración del mercado (que no solo se presenta en el servicio de internet móvil, sino también en el de telefonía móvil) ha impulsado a que desde entes de control como la Comisión de Regulación de Comunicaciones - CRC nazcan iniciativas enfocadas en la protección del usuario ante los operadores móviles, la principal iniciativa se materializó con la Resolución 3066 de 2011, "por la cual se establece el Régimen Integral de Protección de los Derechos de los Usuarios de los Servicios de Comunicaciones", documento que fue compilado por medio de la Resolución 5050 de 2016 y posteriormente actualizado por la Resolución 5111 de 2017, que entró en vigencia desde el 1 . $^{\circ}$ de enero de 2018. Estas últimas actualizaciones a dicho régimen cambiaron drásticamente las reglas de juego en temas clave para los operadores, como lo fue la eliminación de las cláusulas de permanencia, la portabilidad numérica y la simplificación de los contratos.

En cuanto a la satisfacción de los usuarios, el Régimen establece (artículo 2.1.25.7 numeral 2.1.25.7.4 de la Resolución 5111 de 2017) que

Los operadores deberán implementar, medir y publicar a través de los distintos medios de atención, y remitir a la $\mathrm{CRC}$ (...), los resultados de la medición del indicador de satisfacción al usuario respecto de cada uno de los medios de atención, los cuales deben contar con la certificación de un auditor externo y contar con nivel de confianza del $95 \%$.

Aunque el indicador propuesto en esta normativa es ampliamente conocido en la literatura como "Net Promoter Score - NPS" (Reichheld, 2003) y constituye una métrica para la medición de la satisfacción, este solo indaga acerca del nivel de recomendación del usuario respecto al canal de comunicación utilizado y no permite tener una mirada general de la satisfacción del usuario que abarque todos los aspectos y momentos de la prestación del servicio.

Una propuesta metodológica para la medición de la satisfacción de los usuarios debe tener como punto de partida los modelos propuestos para los índices de nacionales de satisfacción que surgieron en los años noventa en Europa y posteriormente se extendieron hacia Asia y Norteamérica (Ospina y Gil, 2011). Estos modelos parten de la definición de satisfacción del cliente propuesta por Kotler (2001), quien considera que dicho concepto está compuesto por tres elementos clave: las expectativas del cliente, el rendimiento percibido del producto o servicio y el nivel de satisfacción; este último como resultado de la interacción de los dos primeros. 
La primera propuesta de modelo de índice nacional de satisfacción fue conocida como Swedish Customer Satisfaction Barometer (Fornell, 1992) y delimitaba un modelo de ecuaciones estructurales en donde la satisfacción definida como variable central se relaciona con un conjunto de variables tanto de antecedentes (expectativas del cliente y rendimiento percibido) como de consecuencias (reclamaciones del cliente y lealtad).

Figura 1. Relación de variables del Swedish Customer Satisfaction Barometer

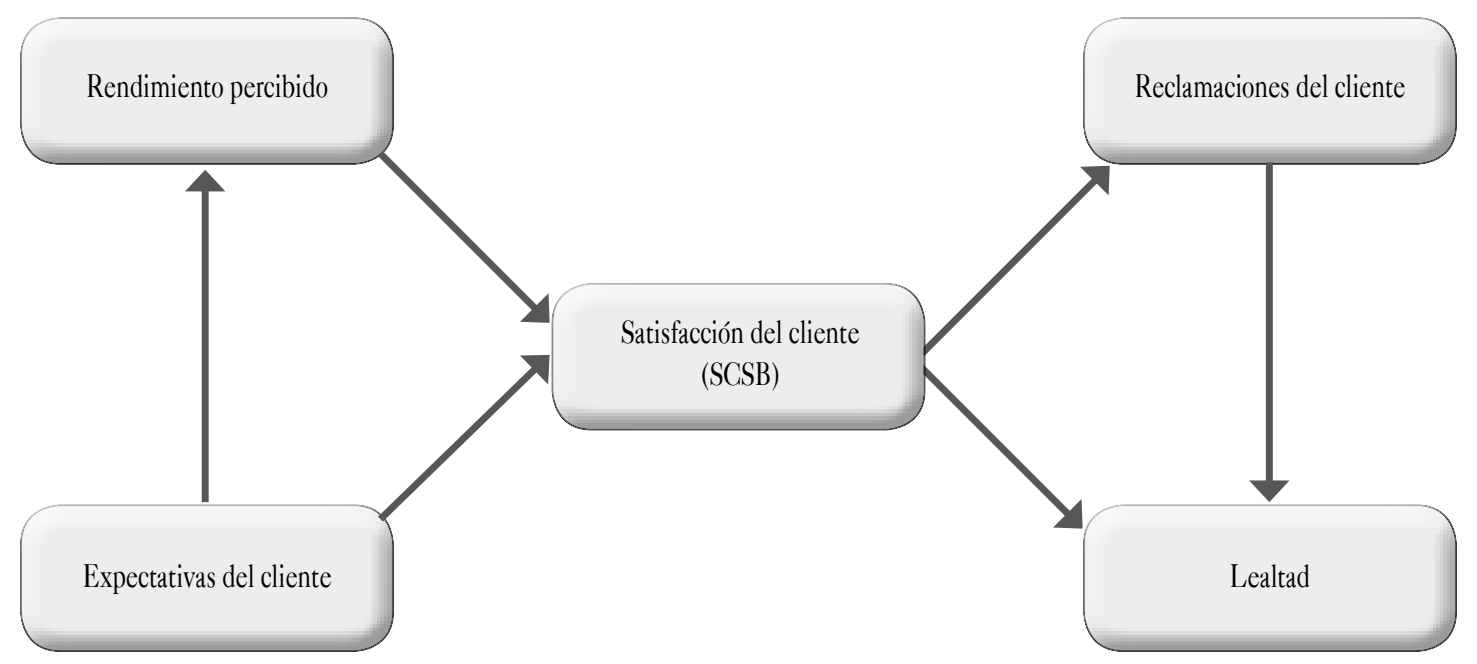

Fuente: Ospina y Gil (2011).

Posteriormente se conocieron otras propuestas como American Customer Satisfaction Index (Fornell, Johnson, Anderson \& Bryant, 1996), European Customer Satisfaction Index (Eklöf, 2000), Norwegian Customer Satisfaction Barometer (Johnson, Gustafsson, Andreassen, Lervik \& Cha, 2001) y Hong Kong Customer Satisfaction Index (Chan et al., 2003), las cuales consistían en modelos más complejos que involucraban más variables como el precio y la imagen corporativa, entre otras. Lo anterior demuestra que para la medición de la satisfacción cada vez es más necesario ahondar en la experiencia del usuario en los diferentes momentos del ciclo de prestación del servicio y considerar más variables que parecen ser relevantes para tal fin.

A nivel regional, el principal antecedente es el Estudio de satisfacción de usuarios de servicios de telecomunicaciones publicado de forma anual por el Ministerio de Transportes y Telecomunicaciones de Chile (2019), el cual para 2018 se realizó con un total de 14092 encuestas telefónicas a nivel nacional en las cuales se evaluaron 5 operadores móviles y 7 prestadores de servicios fijos indagando acerca de la satisfacción en torno a la velocidad del servicio y la atención recibida por los canales disponibles.

En este contexto, la presente investigación propone un modelo metodológico para la medición de la satisfacción de los usuarios de internet móvil, que además de contener elNPS permita medir de una forma representativa y comparable la satisfacción de los usuarios del servicio de internet móvil en las principales ciudades de Colombia, abarcando la experiencia del usuario en todas de las fases del ciclo de prestación del servicio. En el desarrollo de la investigación, se diseñó un cuestionario y un plan 
de procesamiento y análisis para la medición de la satisfacción de los usuarios que fueron aplicados en el año 2016 en una muestra representativa de las principales ciudades de Colombia, lo cual permitió conocer el nivel de satisfacción de los usuarios del servicio de internet móvil prestado por los diferentes operadores a partir de su desempeño en cada uno de los momentos de la prestación del servicio.

Figura 2. Relación de variables del Norwegian Customer Satisfaction Barometer

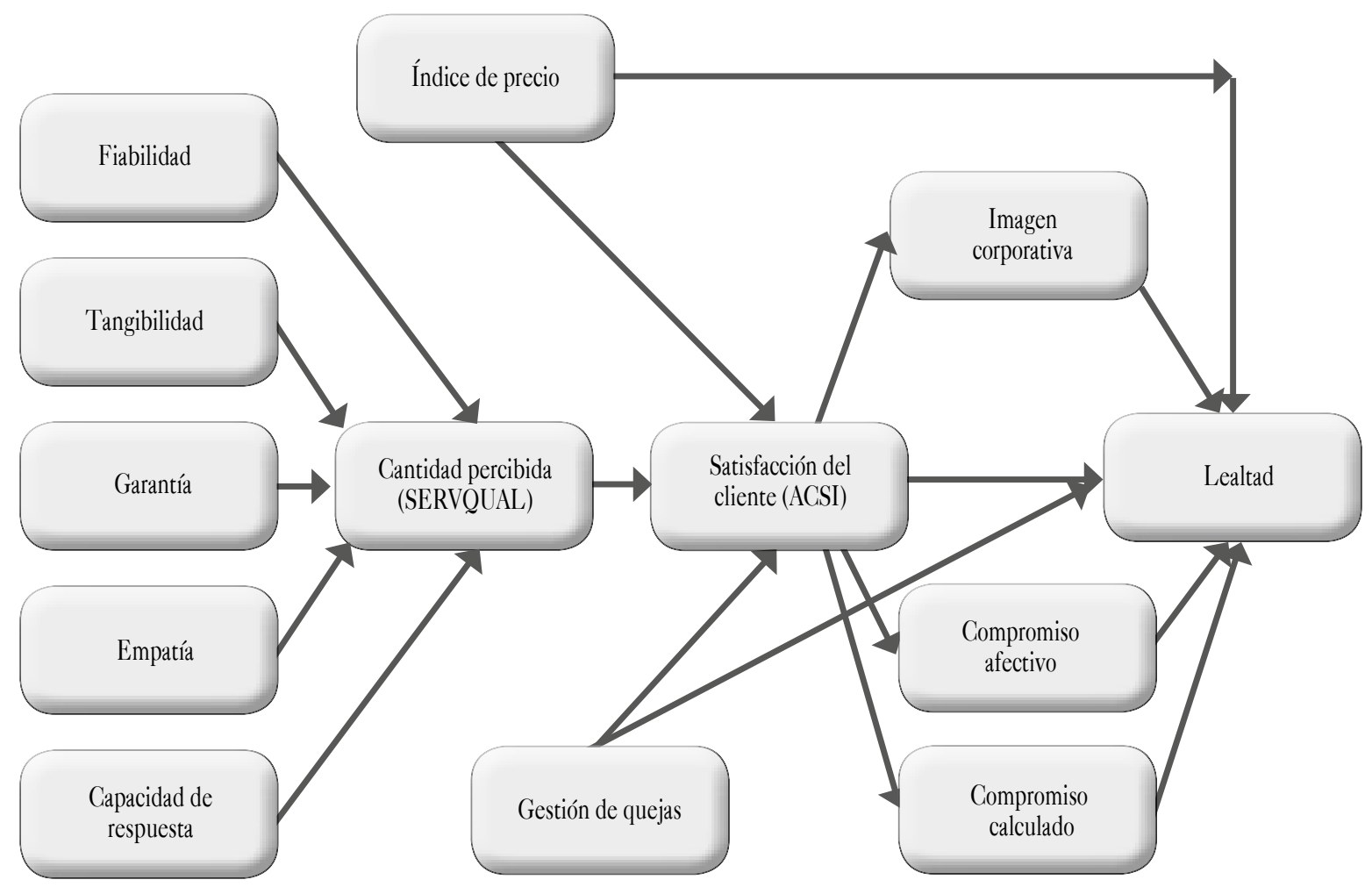

Fuente: Ospina y Gil (2011).

\section{METODOLOGÍA}

Para el logro del objetivo planteado en la investigación se llevó a cabo un proceso de enfoque cuantitativo y alcance correlacional, ya que no solamente pretendía recolectar información que permitiera medir la satisfacción de los usuarios de un servicio, sino que buscaba establecer la relación entre la satisfacción de estos y el desempeño de los procesos del operador en los diferentes momentos del ciclo de prestación del servicio. El desarrollo del estudio fue consecuente con lo planteado por Hernández-Sampieri, Fernández \& Baptista (2014) respecto a que para el desarrollo de este tipo de investigación primero deben medirse las distintas variables para posteriormente analizarlas y establecer las correlaciones entre estas. El proceso desarrollado se describe en la figura 3.

El modelo teórico sobre el cual se propone el desarrollo metodológico abarca 7 constructos identificados como relevantes para explicar la satisfacción de los usuarios y 2 adicionales, que propone el autor, como resultado de la interacción de los primeros. 
Figura 3. Ejecución para la medición de la satisfacción de los usuarios de internet móvil

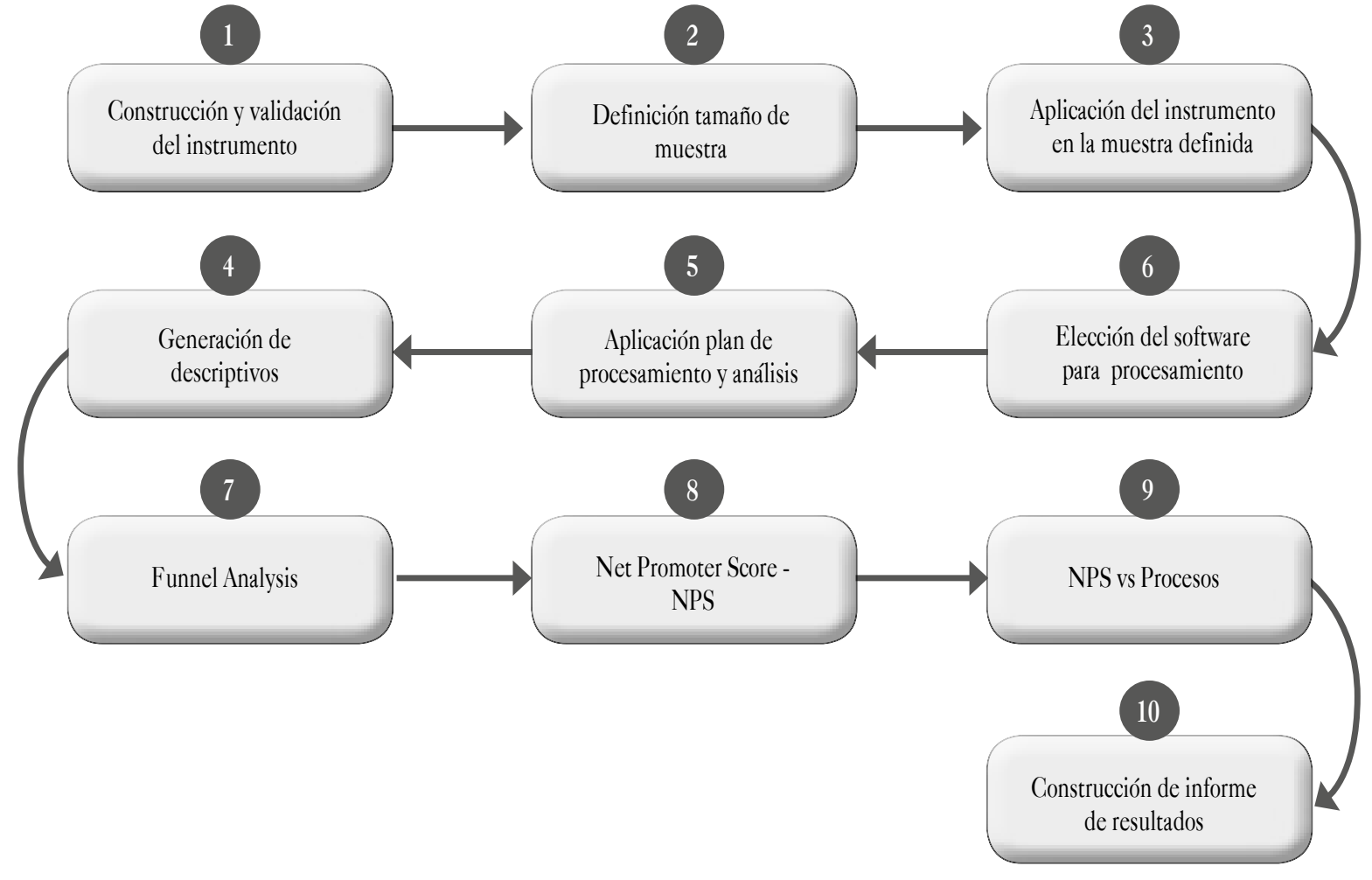

Fuente: elaboración propia.

Figura 4. Modelo teórico de la satisfacción de usuarios de internet móvil en Colombia

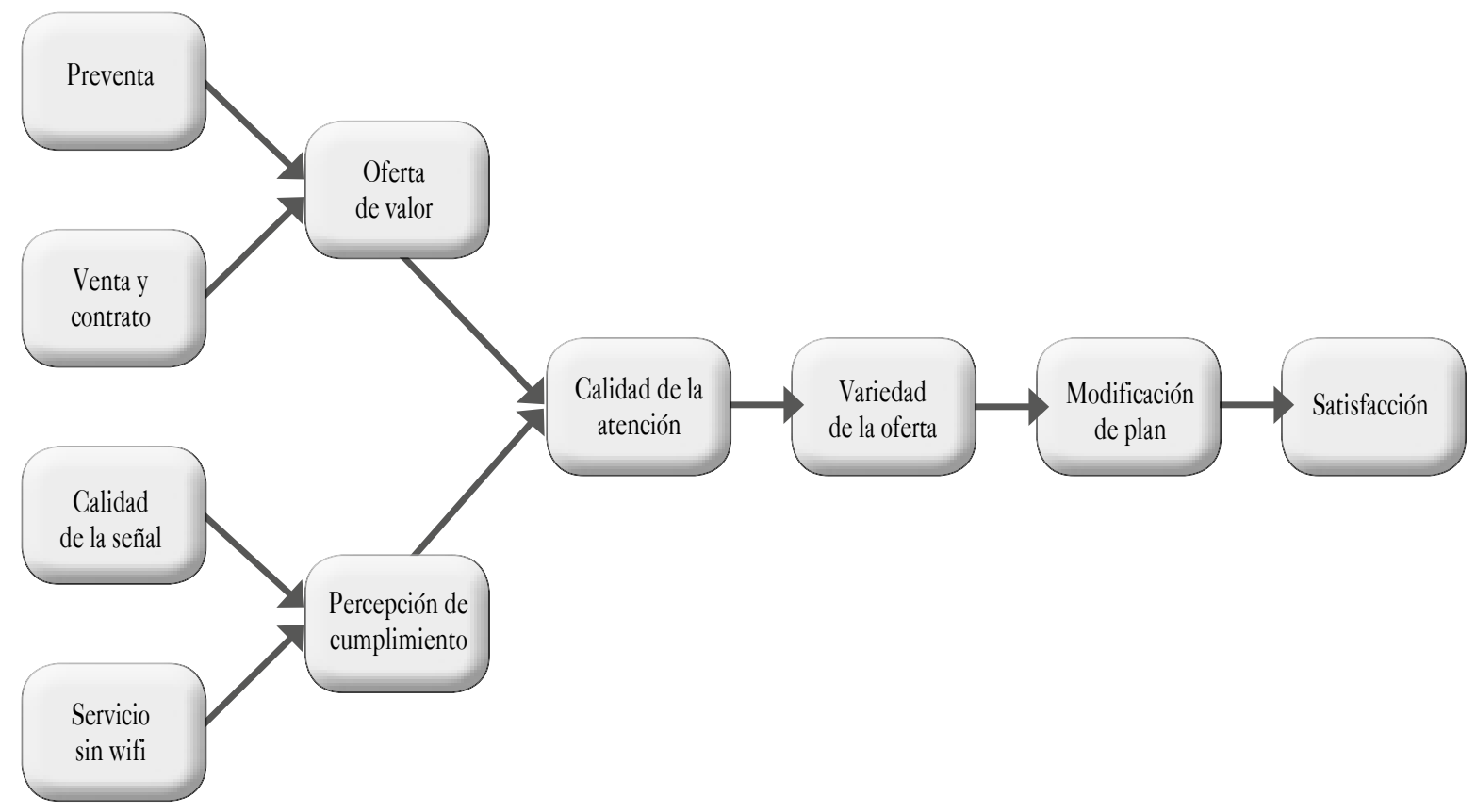

Fuente: elaboración propia. 
El modelo parte de la atención preventa y el proceso de venta y firma de contrato del servicio, momentos en los cuales se genera toda la expectativa frente a la oferta de valor que se le está haciendo al futuro usuario. Ya una vez contratado el servicio el usuario construye su percepción de cumplimiento a partir de la calidad de la señal y el desempeño del servicio sin estar conectado a una red wifi.

En la mediación entre esa expectativa de oferta de valor y la percepción de cumplimiento se encuentra la calidad de la atención que el usuario recibe por los canales dispuestos por su operador por medio de los cuales, y apalancado en la variedad de la oferta, el operador atiende los requerimientos del usuario con el fin de corregir y adaptar las características del servicio a las necesidades del usuario. De la efectividad de estas acciones y de la facilidad para aplicar las modificaciones se impacta en gran medida la satisfacción del usuario.

\section{Construcción y validación del instrumento}

A partir del know how de una agencia de investigación de mercados, las discusiones y material socializado en diferentes eventos enmarcados en el proyecto regulatorio "Revisión Integral del Régimen de Protección de Usuarios de los Servicios de Comunicaciones", se establecieron 7 áreas funcionales a partir de las cuales se desarrolló el instrumento de investigación: comercial, canales, producto, precio, upgrade, cancelación y promoción. A partir de estas áreas funcionales y sus procesos se diseñaron inicialmente 36 preguntas divididas de la siguiente forma: 7 preguntas básicas de caracterización, 1 pregunta NPS y 28 preguntas exploratorias relacionadas con la experiencia del usuario en todo el ciclo de prestación del servicio de internet móvil.

Tabla 1. Descripción preguntas básicas de caracterización

\begin{tabular}{|l|}
\hline \multicolumn{1}{|c|}{ Ítem } \\
\hline $\begin{array}{l}\text { ¿Qué tipo de terminal móvil (celular) usted posee en este } \\
\text { momento }\end{array}$ \\
\hline $\begin{array}{l}\text { ¿Actualmente cuenta con un plan de internet móvil para su } \\
\text { celular? }\end{array}$ \\
\hline ¿Hace cuánto contrato usted este plan de internet móvil? \\
\hline $\begin{array}{l}\text { ¿Cuáles operadores móviles conoce así nunca haya contratado un } \\
\text { servicio con ellos? }\end{array}$ \\
\hline $\begin{array}{l}\text { ¿Con cuales operadores móviles ha contratado alguna vez algún } \\
\text { servicio? }\end{array}$ \\
\hline ¿Cuál es su operador móvil actual? \\
\hline $\begin{array}{l}\text { ¿Cuál o cuáles operadores móviles nunca contrataría ni } \\
\text { recomendaría? }\end{array}$ \\
\hline
\end{tabular}

Fuente: elaboración propia.

Tabla 2. Descripción pregunta NPS

\begin{tabular}{|l|}
\hline \multicolumn{1}{c|}{ Ítem } \\
\hline $\begin{array}{l}\text { ¿Recomendaría el servicio de internet móvil de su operador a otra } \\
\text { persona? }\end{array}$ \\
\hline
\end{tabular}

Fuente: elaboración propia. 
Tabla 3. Descripción preguntas por área funcional

\begin{tabular}{|c|c|}
\hline Área funcional & Ítems \\
\hline \multirow{2}{*}{ General } & En términos generales, considero que mi operador es \\
\hline & Respecto a lo que me prometieron al momento de ofrecerme el servicio y lo que he recibido, mi operador es \\
\hline \multirow{7}{*}{ Comercial } & Respecto a la atención preventa, mi operador es \\
\hline & Respecto a la rapidez y claridad del proceso de venta, mi operador es \\
\hline & Respecto a la claridad del contrato, mi operador es \\
\hline & Respecto a la cantidad de alterativas (canales) de comunicación que haya a mi disposición, mi operador es \\
\hline & Respecto a la calidad de la atención del personal cuando me comunico por cualquiera de los canales, mi operador es \\
\hline & Respecto al procedimiento para la resolución de solicitudes, peticiones quejas y reclamos, mi operador es \\
\hline & Respecto a la prontitud con la que atienden y responden mis solicitudes, mi operador es \\
\hline \multirow{5}{*}{ Canales } & Respecto a la funcionalidad (rapidez, calidad de la atención e información) del call center, mi operador es \\
\hline & $\begin{array}{l}\text { Respecto a la funcionalidad (rapidez, calidad de la atención e información) de los puntos de atención presencial, mi } \\
\text { operador es }\end{array}$ \\
\hline & Respecto a la funcionalidad (rapidez, calidad de la atención e información) de la página web, mi operador es \\
\hline & Respecto a los horarios de atención de los canales de comunicación, mi operador es \\
\hline & Respecto a la calidad y estabilidad de la señal para el servicio de datos móviles, mi operador es \\
\hline \multirow{3}{*}{ Producto } & Respecto a la velocidad del servicio de datos móviles, mi operador es \\
\hline & Respecto a la cobertura del servicio de datos móviles, mi operador es \\
\hline & Respecto a la tarifa cobrada por el servicio de datos móviles, mi operador es \\
\hline \multirow{3}{*}{ Precio } & Respecto a la puntualidad y claridad de la factura, mi operador es \\
\hline & Respecto a los medios de pago disponibles, mi operador es \\
\hline & Respecto a los métodos de cobro por retraso o falta de pago, mi operador es \\
\hline \multirow{2}{*}{ Upgrade } & Respecto a la facilidad del proceso para modificar mi plan de datos, mi operador es \\
\hline & Respecto al costo e implicaciones (penalidades, multas) que tiene cambiar mi plan de datos, mi operador es \\
\hline \multirow{2}{*}{ Cancelación } & Respecto a la facilidad del proceso para cancelar mi plan de datos, mi operador es \\
\hline & Respecto al costo e implicaciones (penalidades, multas) que tiene cambiar mi plan de datos, mi operador es \\
\hline \multirow{4}{*}{ Promoción } & Respecto a las promociones en planes de datos móviles, mi operador es \\
\hline & Respecto a la oferta de planes de internet móvil, mi operador es \\
\hline & Respecto a la oferta de equipos móviles, mi operador es \\
\hline & Respecto a los medios que utiliza para comunicar planes y promociones, mi operador es \\
\hline
\end{tabular}

Fuente: elaboración propia. 
En cuanto a las alternativas de respuestas para los 28 ítems exploratorios, se propuso una escala de 3 opciones:

Tabla 4. Opciones de respuesta

\begin{tabular}{|l|}
\hline \multicolumn{1}{|c|}{ Opción respuestas } \\
\hline Peor que los demás operadores \\
\hline Igual que los demás operadores \\
\hline Mejor que los demás operadores \\
\hline
\end{tabular}

Fuente: elaboración propia.

Para determinar la escala propuesta anteriormente el autor se basó en los postulados de Hunt (Velandia, Ardón \& Jara, 2007), en los cuales se propone que la percepción de satisfacción de un usuario de un producto o servicio, aparte de basarse en el desempeño funcional de este, toma como referencia el desempeño observado de los mismos servicios en otros usuarios; es decir, que el nivel de satisfacción de un usuario con su operador refleja el desempeño observado por este usuario en los operadores contratados por sus amigos y familiares.

Para efectos del presente estudio, la consideración por parte del usuario de que el desempeño de su operador actual es "peor que los demás operadores" denota insatisfacción con el servicio, pues se encuentra por debajo del nivel esperado y del desempeño de los demás.

La consideración de que el desempeño es "igual que los demás operadores" denota un nivel de satisfacción aceptable, es decir, que el servicio cumple con el mínimo desempeño esperado y no se diferencia del desempeño de los demás.

La consideración de que el desempeño es "mejor que los demás operadores" denota una satisfacción superior, el servicio presenta un desempeño superior a lo esperado incluso por encima del desempeño de los demás.

El instrumento desarrollado fue configurado en un formato de cuestionario estándar y se procedió a realizar dos validaciones sobre este.

La primera validación corrió por cuenta de 7 jueces expertos cuyo objetivo fue validar el contenido del instrumento, es decir, que el conjunto de ítems respondiera a las necesidades de la investigación y que cada uno de ellos midiera lo que se planeó medir. Estos expertos cuentan con una amplia trayectoria en los ámbitos de las telecomunicaciones, la estadística, la investigación de mercados y el estudio del comportamiento del consumidor.

Esta etapa de la investigación tuvo una duración de 45 dís aproximadamente contados a partir de la remisión del instrumento a cada uno de ellos hasta la recepción de sus observaciones. Con los resultados de la evaluación por jueces expertos se realizaron cambios y ajustes sugeridos al cuestionario.

La segunda validación tuvo como objetivo comprobar que los términos utilizados y la redacción del instrumento resultaran entendibles y fuera fácil para el usuario navegar en este. Tal validación se realizó mediante la estructuración del cuestionario en una plataforma web y su posterior envío al correo electrónico de los participantes. En total, se contó con la participación de 30 usuarios a los cuales, tras el registro de la respuesta de cada pregunta, se les preguntaba si esta había sido clara y contenía un campo para registrar observaciones al respecto.

El cuestionario definitivo quedó compuesto por las siguientes 50 preguntas: 
Tabla 5. Descripción preguntas cuestionario definitivo

\begin{tabular}{|c|c|}
\hline Área funcional & Ítems \\
\hline \multirow{10}{*}{ General } & ¿Qué tipo de teléfono celular usted posee en este momento? \\
\hline & ¿Actualmente cuenta con un plan de internet móvil para su celular? \\
\hline & ¿Hace cuánto contrato usted ese plan de internet móvil? \\
\hline & ¿Cuáles operadores de telefonía e internet móvil conoce así nunca haya contratado un servicio con ello? \\
\hline & ¿Con cuáles operadores de telefonía e internet móvil ha contratado alguna vez algún servicio? \\
\hline & ¿Cuál es el operador que le presta el servicio de internet móvil actualmente? \\
\hline & ¿Cuál será su próximo operador del servicio de internet móvil? \\
\hline & ¿Cuál o cuáles operadores de telefonía móvil nunca contrataría mi recomendaría? \\
\hline & En una escala de 1 a 10, siendo 1 "nunca lo recomendaría" y 10 "definitivamente lo recomendaría" \\
\hline & Los demás piensan que mi operador es \\
\hline \multirow{5}{*}{ Comercial } & Respecto a lo que me prometieron al momento de ofrecerme el servicio y lo que he recibido \\
\hline & Respecto a la atención preventa (mientras averiguaba y elegia), mi operador es \\
\hline & Respecto a la rapidez del proceso de venta, mi operador es \\
\hline & Respecto a la claridad del proceso de venta, mi operador es \\
\hline & Respecto a la claridad del contrato, mi operador es \\
\hline \multirow{9}{*}{ Canales } & Respecto a la cantidad de canales de comunicación que hay a mi disposición, mi operador es \\
\hline & Respecto a la calidad de atención del personal cuando me comunicó por cualquiera de los canales, mi operador tiene \\
\hline & Respecto al procedimiento para la resolución de solicitudes, peticiones, quejas y reclamos, mi operador es \\
\hline & Respecto a la prontitud con la que atienden y responden mis solicitudes, mi operador es \\
\hline & Respecto a la funcionalidad (rapidez, calidad de la atención e información) del call center, mi operador es \\
\hline & Respecto a la funcionalidad (rapidez, calidad de la atención e información) de los puntos de atención presencial, mi operador es \\
\hline & web, mi operador es \\
\hline & Respecto a la funcionalidad (rapidez, calidad de la atención e información) de la página, mi operador es \\
\hline & Respecto a los horarios de atención de los canales de comunicación, mi operador es \\
\hline \multirow{9}{*}{ Producto } & Respecto a la calidad y estabilidad de la señal para el servicio de datos móviles, mi operador es \\
\hline & Respecto a la velocidad del servicio de datos móviles, mi operador es \\
\hline & Respecto a la cobertura del servicio de datos móviles, mi operador es \\
\hline & Respecto a la calidad de la señal para el servicio de internet móvil, mi operador es \\
\hline & Respecto a la estabilidad de la señal para el servicio de internet móvil, mi operador es \\
\hline & Respecto a la velocidad de subida (al cargar fotos y archivos) del servicio de internet móvil, mi operador es \\
\hline & Respecto a la cobertura geográfica del servicio de internet móvil, mi operador es \\
\hline & El desempeño de mi operador para dejarme ver mi correo electrónico sin estar conectado a wifi es \\
\hline & $\begin{array}{l}\text { El desempeño de mi operador para dejarme utilizar aplicaciones de mensajería instantánea como WhatsApp sin estar } \\
\text { conectado a wifi es }\end{array}$ \\
\hline
\end{tabular}




\begin{tabular}{|c|c|}
\hline Área funcional & Ítems \\
\hline \multirow{3}{*}{ Producto } & El desempeño de mi operador para dejarme ver videos on-line en aplicaciones como YouTube sin estar conectado a wifi es \\
\hline & El desempeño de mi operador para dejarme subir y mandar fotos sin estar conectado a wifi es \\
\hline & El desempeño de mi operador para dejarme ver páginas web sin estar conectado a wifi es \\
\hline \multirow{4}{*}{ Precio } & Respecto a la tarifa cobrada por el servicio de internet móvil, mi operador es \\
\hline & Respecto a la puntualidad de la factura, mi operador es \\
\hline & Respecto a la claridad de la factura, mi operador es \\
\hline & Respecto a los medios de pago disponibles, mi operador tiene \\
\hline \multirow{2}{*}{ Upgrade } & Respecto a la facilidad del proceso para modificar mi plan de internet móvil, mi operador es \\
\hline & Respecto al costo e implicaciones (penalidades, multas) que tiene cambiar mi plan de internet móvil, mi operador es \\
\hline \multirow[b]{2}{*}{ Cancelación } & Respecto a la facilidad del proceso para cancelar (dar por terminado) mi plan de internet móvil, mi operador es \\
\hline & $\begin{array}{l}\text { Respecto al costo e implicaciones (penalidades, multas) que tiene cancelar (dar por terminado) mi plan de internet móvil, } \\
\text { mi operador es }\end{array}$ \\
\hline \multirow{8}{*}{ Promoción } & Respecto a la oferta y variedad de planes de internet móvil, mi operador tiene \\
\hline & Respecto a las promociones para planes de internet móvil, mi operador es \\
\hline & Respecto a la oferta de equipos móviles, mi operador tiene \\
\hline & Respecto a los medios que utiliza para comunicar planes y promociones, mi operador es \\
\hline & La publicidad en internet de mi operador móvil es \\
\hline & La publicidad en radio de mi operador móvil es \\
\hline & La publicidad en televisión de mi operador móvil es \\
\hline & La publicidad en redes sociales de mi operador móvil es \\
\hline
\end{tabular}

Fuente: elaboración propia.

\section{Definición del tamaño y distribución de la muestra}

El cálculo de la muestra representativa para los usuarios de internet móvil en Colombia aplicada en el levantamiento de la información se realizó contemplando un universo de usuarios de 5170000 según lo reportado por la CRC (2018) para el año 2014 y una penetración del internet móvil que, según MinTIC (2018), alcanzó el
19\% para el cierre de 2013. Bajo un escenario de muestreo no probabilístico, por cuotas, respetando un margen de error del $5 \%$ y una confiabilidad del $95 \%$, la estimación muestral con mejor potencia fue 804 entrevistas. Las estimaciones muestrales se efectuaron mediante el software JMP.

Bajo un muestreo representativo, se propuso la siguiente distribución para la muestra: 
Tabla 6. Distribución de la muestra.

\begin{tabular}{|c|c|c|c|c|c|c|}
\hline \multirow{2}{*}{ NSE } & \multirow{2}{*}{ Género } & \multirow{2}{*}{ Edad } & \multicolumn{4}{|c|}{ Ciudad } \\
\hline & & & Bogotá & Medellín & Cali & Barranquilla \\
\hline \multirow{6}{*}{ NSE 2-3 } & \multirow{3}{*}{ Hombre } & 18-25 años & 42 & 24 & 14 & 14 \\
\hline & & 26 -33 años & 42 & 24 & 14 & 14 \\
\hline & & 34-45 años & 42 & 24 & 14 & 14 \\
\hline & \multirow{3}{*}{ Mujer } & 18-25 años & 42 & 24 & 14 & 14 \\
\hline & & $26-33$ años & 42 & 24 & 14 & 14 \\
\hline & & 34-45 años & 42 & 24 & 14 & 14 \\
\hline \multirow{6}{*}{ NSE 4-5 } & \multirow{3}{*}{ Hombre } & 18-25 años & 18 & 10 & 6 & 6 \\
\hline & & 26 -33 años & 18 & 10 & 6 & 6 \\
\hline & & 34-45 años & 18 & 10 & 6 & 6 \\
\hline & \multirow{3}{*}{ Mujer } & 18-25 años & 18 & 10 & 6 & 6 \\
\hline & & 26 -33 años & 18 & 10 & 6 & 6 \\
\hline & & 34-45 años & 18 & 10 & 6 & 6 \\
\hline
\end{tabular}

Fuente: elaboración propia.

Se controlaron cuotas mediante el cruce de celdas para las variables de género, rango de edad, ciudad y nivel socioeconómico (NSE); las dos últimas aportan la proporcionalidad de la distribución respecto a filas y columnas, es decir, se mantiene la proporcionalidad entre la distribución real de la población y la distribución de la muestra en cuanto al NSE y en cuanto a población de las ciudades estudiadas.

\section{Aplicación del instrumento en la muestra definida}

La aplicación del cuestionario se llevó a cabo sobre la distribución muestral desarrollada para las 4 principales ciudades de Colombia en el mes de septiembre del año 2016, mediante la técnica de panel auditado online. Estos paneles auditados son comunidades on-line conformadas por personas predispuestas a participar en proyectos de investigación de mercados (en su mayoría encuestas) a cambio de alguna compensación como dinero, obsequios o sorteos.

\section{Elección del software para procesamiento de los datos}

Para este ejercicio, se solicitó al proveedor una base de datos en formato compatible con el software IBM SPSS (Statistical Package for the Social Sciences o Paquete Estadístico para las Ciencias Sociales). Este paquete de herramientas estadísticas es ampliamente reconocido y recomendado por investigadores y autores como Hernández-Sampieri et al. (2014) y Babbie, Wagner \& Zaino (2018); fue desarrollado en la Universidad de Chicago y actualmente es propiedad de IBM. 


\section{Aplicación del plan de procesamiento} $\mathrm{y}$ análisis

Una vez realizado el alistamiento de la base de datos en el software se procedió a ejecutar el plan de procesamiento definido con el fin de generar los tabulados que serán objeto de análisis y con base en los cuales se generarán los gráficos e informes necesarios para la posterior divulgación de los resultados de la medición realizada. Una vez generados los diferentes tabulados en la ventana de visualización de resultados de IBM SPSS se exportan en formato Excel para facilitar su edición y posterior uso.

\section{Generación de descriptivos}

El análisis descriptivo de la información permitió validar el banner demográfico de la muestra, el cual arrojó los siguientes recuentos:

Tabla 7. Distribución de la muestra recolectada

\begin{tabular}{|c|c|c|c|}
\hline & & \\
\hline & & Recuento & $\begin{array}{c}\% \text { del N de la } \\
\text { columna }\end{array}$ \\
\hline \multirow{5}{*}{ Ciudad_R } & Total & 842 & 100 \\
\hline & Bogotá & 381 & 45 \\
\hline & Cali & 174 & 21 \\
\hline & Medellín & 155 & 18 \\
\hline & Barranquilla & 131 & 16 \\
\hline \multirow{2}{*}{ Génereo } & Hombre & 413 & 49 \\
\hline & Mujer & 429 & 51 \\
\hline \multirow{5}{*}{ Edad_R } & $0 \_17$ & 0 & 0 \\
\hline & 18_25 & 258 & 31 \\
\hline & 26_33 & 289 & 34 \\
\hline & $34 \_45$ & 295 & 35 \\
\hline & $46 \_+$ & 0 & 0 \\
\hline \multirow{2}{*}{ NSE_Reco } & NSE_2-3 & 566 & 67 \\
\hline & NSE_4-5 & 276 & 33 \\
\hline
\end{tabular}

Fuente: elaboración propia.
El comportamiento de las variables extraídas, aunque difiere un poco del diseño muestral propuesto, asegura la consistencia y representatividad de la información. Estas desviaciones se deben a la dificultad que tuvo el proveedor para completar la muestra requerida en cada una de las celdas del diseño muestral y fueron compensadas con una mayor cantidad de encuestas efectivas. Mediante muestreo por conveniencia en panel auditado on-line se obtuvieron 842 entrevistas dispersas de la siguiente manera: Bogotá (45\%), Cali (21\%), Medellín (18\%) y Barranquilla (16\%). En cuanto al género y la edad de los encuestados se observa una distribución aceptablemente proporcional; y el nivel socioeconómico se encuentra distribuido $67 \%$ estratos $2-3$ y $33 \%$ estratos $4-5$.

En cuanto a la participación por operador, el $43 \%$ de la muestra reporta a Claro como su operador actual, seguido por Movistar y Tigo con 25\% y 22\%, respectivamente. El 10\% restante se distribuye entre el resto de operadores.

Tabla 8. Distribución de la muestra recolectada por operador

\begin{tabular}{|c|c|c|}
\cline { 2 - 3 } \multicolumn{1}{c|}{} & Recuento & \% de N columna \\
\hline Total & 842 & 100 \\
\hline Claro & 358 & 43 \\
\hline Movistar & 214 & 25 \\
\hline Tigo & 186 & 22 \\
\hline Virgin Mobile & 29 & 3 \\
\hline ETB & 28 & 3 \\
\hline Avantel & 14 & 2 \\
\hline Móvil Éxito & 6 & 1 \\
\hline Uff Móvil & 4 & 0 \\
\hline UNE-EPM & 3 & 0 \\
\hline
\end{tabular}

Fuente: elaboración propia. 


\section{Funnel Analysis}

Análisis enfocado en el desempeño general del operador en el mercado, mide el conocimiento de la marca, quiénes lograron un primer uso y cuántos de estos son usuarios actuales. Se construyó con las respuestas de las preguntas 4,5 y 6 del cuestionario.

1. ¿Cuáles operadores de telefonía e internet móvil conoce, así nunca haya contratado servicios con ellos?

2. ¿Con cuáles operadores de telefonía e internet móvil ha contratado alguna vez algún servicio?

3. ¿Cuál es el operador que le presta el servicio de internet móvil actualmente?

\section{Net Promoter Score - NPS}

Este indicador, ampliamente utilizado en la medición de la satisfacción de clientes (Laitinen, 2018), se calculó empleando la pregunta 9 del cuestionario en la cual se indaga el nivel de recomendación del operador actual reportado por cada usuario.

Con base en la calificación reportada en dicha pregunta se agruparon los usuarios según se muestra en la tabla 9.

Tabla 9. Clasificación de usuarios según calificación NPS

\begin{tabular}{|c|c|}
\hline Calificación & Identificación \\
\hline Entre 9 y 10 & Promotores \\
\hline Entre 7 y 8 & Pasivos \\
\hline Entre 1 y 6 & Detractores \\
\hline
\end{tabular}

Fuente: elaboración propia.
Posteriormente el indicador se obtiene como resultado de restar el porcentaje de detractores al porcentaje de promotores, así:

$$
\text { NPS }=\left(\frac{\text { Promotores }}{\text { Total Encuestados }} \times 100\right)-\left(\frac{\text { Detractores }}{\text { Total Encuestados }} \times 100\right)
$$

\section{Análisis NPS contra el desempeño del operador en los diferentes aspectos y momentos del ciclo de prestación del servicio}

Una vez se realizó el cálculo del indicador NPS, se procedió a desarrollar un análisis que permite indagar acerca de la relación entre el nivel de recomendación reportado en el NSP y el desempeño del operador en las diferentes preguntas del cuestionario.

Este análisis permitió identificar debilidades y fortalezas en diversos aspectos para cada operador con base en las cuales cada uno podría implementar planes de acción para mejorar la satisfacción del usuario en dicho aspecto e impactar de forma positiva su indicador de NPS.

\section{RESULTADOS Y DISCUSIÓN}

En los resultados del Funnel Analysis se puede observar que los tres operadores más grandes reportan los niveles más altos de conocimiento de la marca, primer uso y uso actual. 
Tabla 10. Funnel Analysis por operador

\begin{tabular}{|l|c|c|c|}
\cline { 2 - 4 } \multicolumn{1}{c|}{} & P4 & P5 & P6 \\
\cline { 2 - 4 } \multicolumn{1}{c|}{} & Conocimiento & Primer uso & Uso actual \\
\hline Total & 100 & 100 & 100 \\
\hline Movistar & $70 \%$ & $47 \%$ & $25 \%$ \\
\hline Tigo & $70 \%$ & $42 \%$ & $22 \%$ \\
\hline Avantel & $44 \%$ & $4 \%$ & $2 \%$ \\
\hline Virgin Mobile & $54 \%$ & $8 \%$ & $3 \%$ \\
\hline Uff Móvil & $48 \%$ & $4 \%$ & $0,5 \%$ \\
\hline ETB & $47 \%$ & $9 \%$ & $3 \%$ \\
\hline Móvil Éxito & $39 \%$ & $3 \%$ & $1 \%$ \\
\hline UNE-EPM & $33 \%$ & $6 \%$ & $0,4 \%$ \\
\hline
\end{tabular}

Fuente: elaboración propia.

En el caso de Claro, el 78\% de la muestra reporta conocer la marca, de estos el $64 \%$ lo usó por primera vez y el $43 \%$ lo mantiene como su operador actual. Movistar y Tigo reportan un nivel de conocimiento de marca similar con el $70 \%$ de la muestra, pero en el caso del primero el $47 \%$ reportaron primer uso y el $25 \%$ como operador actual. En el caso de Tigo, los porcentajes fueron $42 \%$ y $22 \%$, respectivamente.

A partir de este análisis los operadores pueden desarrollar planes de acción que les permitan mejorar su desempeño en los diferentes niveles. Por ejemplo, si un operador como Móvil Éxito quisiera mejorar su indicador de conocimiento (39\%) podría desarrollar estrategias alrededor de campañas de comunicación y branding. Si, por el contrario, su objetivo es incrementar el primer uso, como el caso de Avantel, podría implementar promociones para nuevos usuarios y facilidades para el cambio de operador. Para mejorar el último nivel correspondiente al uso actual, es clave el desempeño de la prestación del servicio de tal forma que el usuario esté satisfecho y se retenga.
Respecto a los operadores evaluados, el mayor indicador de NPS lo obtuvo el Virgin Mobile (55\%) superando con amplia ventaja a ETB y Avantel (ambos con NPS del 29\%). El cuarto y quinto lugar los ocupan Tigo (23\%) y Movistar (14\%), respectivamente. Esto quiere decir que los usuarios más satisfechos y quienes con una alta probabilidad recomendarían son los de Virgin Mobile.

Por el contrario, UFF Móvil y UNE-EPM reportan un nivel de recomendación nulo, mientras que los niveles de recomendación son negativos para Claro (-10\%) y Móvil Éxito (-17\%).

Tabla 11. Resultados NPS por operador

\begin{tabular}{|l|c|}
\cline { 2 - 2 } \multicolumn{1}{c|}{} & NPS \\
\hline Virgin Mobile & $55 \%$ \\
\hline ETB & $29 \%$ \\
\hline Avantel & $29 \%$ \\
\hline Tigo & $23 \%$ \\
\hline Movistar & $14 \%$ \\
\hline Uff Móvil & $0 \%$ \\
\hline UNE-EPM & $0 \%$ \\
\hline Claro & $-10 \%$ \\
\hline Móvil Éxito & $-17 \%$ \\
\hline
\end{tabular}

Fuente: elaboración propia.

$\mathrm{Al}$ analizar a nivel general los resultados del NPS contra el desempeño de los operadores en todo el ciclo de prestación del servicio, se observan cuatro aspectos que están impactando significativamente de forma negativa el nivel de recomendación de los operadores de internet móvil:

- Cumplimiento de la promesa de venta: con un impacto del -27\% el incumplimiento de lo que le 
prometieron al usuario al momento de contratar el servicio es el aspecto que más deteriora el NPS de los operadores.

- Claridad del proceso de venta: con un impacto del $-27 \%$, encontrarse frente a procesos poco claros o confusos para la contratación del servicio es el segundo aspecto que más deteriora el nivel de recomendación por parte de los usuarios.
- Rapidez del proceso de venta: con un impacto del $-25 \%$ sobre el NPS, los procesos lentos y que conllevan gran cantidad de trámites o tiempos de espera, son el tercer aspecto que deteriora este indicador.

- Horarios de atención de los canales de comunicación: al igual que el aspecto anterior con un impacto del $-25 \%$, los horarios poco flexibles frente a las rutinas de los usuarios son el cuarto aspecto que más impacta negativamente el NPS.

Tabla 12. Análisis impacto del desempeño en el NPS

\begin{tabular}{|c|c|c|}
\hline \multirow{2}{*}{ Aspecto del ciclo de presentación del servicio evaluado } & \multicolumn{2}{|c|}{ Impacto } \\
\hline & Peor & Mejor \\
\hline Cumplimiento de la promesa de venta & $-27 \%$ & $22 \%$ \\
\hline Atención preventa (mientras averiguaba y elegia) & $-22 \%$ & $8 \%$ \\
\hline Rapidez del proceso de venta & $-25 \%$ & $8 \%$ \\
\hline Claridad del proceso de venta & $-27 \%$ & $13 \%$ \\
\hline Claridad del contrato & $-18 \%$ & $11 \%$ \\
\hline Cantidad de canales de comunicación a disposición del usuario & $-15 \%$ & $8 \%$ \\
\hline Calidad de la atención por medio de los canales de comunicación & $-22 \%$ & $11 \%$ \\
\hline Procedimiento para resolución de PQRS & $-18 \%$ & $11 \%$ \\
\hline Prontitud de la atención y respuesta & $-16 \%$ & $13 \%$ \\
\hline Horarios de atención de los canales de comunicación & $-25 \%$ & $9 \%$ \\
\hline Calidad de la señal para el servicio & $-21 \%$ & $10 \%$ \\
\hline Estabilidad de la señal para el servicio & $-20 \%$ & $11 \%$ \\
\hline Velocidad de subida del servicio & $-22 \%$ & $12 \%$ \\
\hline Velocidad de baja del servicio & $-21 \%$ & $12 \%$ \\
\hline Cobertura geográfica del servicio & $-12 \%$ & $5 \%$ \\
\hline Desempeño del servicio en e-mail sin conexión wifi & $-11 \%$ & $13 \%$ \\
\hline Desempeño del servicio en redes sociales sin conexión wifi & $-17 \%$ & $11 \%$ \\
\hline Desempeño del servicio en la visualización de videos sin conexión wifi & $-11 \%$ & $15 \%$ \\
\hline
\end{tabular}




\begin{tabular}{|c|c|c|}
\hline \multirow{2}{*}{ Aspecto del ciclo de presentación del servicio evaluado } & \multicolumn{2}{|c|}{ Impacto } \\
\hline & Peor & Mejor \\
\hline Desempeño del servicio para subir y enviar archivos sin conexión wifi & $-13 \%$ & $13 \%$ \\
\hline Desempeño del servicio para visualizar de páginas web sin conexión wifi & $-14 \%$ & $14 \%$ \\
\hline Tarifa cobrada por el servicio & $4 \%$ & $-3 \%$ \\
\hline Puntualidad de la factura & $-11 \%$ & $-1 \%$ \\
\hline Claridad de la factura & $-12 \%$ & $9 \%$ \\
\hline Medios de pago disponibles & $-18 \%$ & $3 \%$ \\
\hline Facilidad para modificar el plan contratado & $-15 \%$ & $7 \%$ \\
\hline Costos e implicaciones para el cambio del plan contratado & $-18 \%$ & $7 \%$ \\
\hline Facilidad para la cancelación del plan contratado & $-12 \%$ & $12 \%$ \\
\hline Costos e implicaciones de la cancelación de plan contratado & $-14 \%$ & $11 \%$ \\
\hline Oferta y variedad de planes disponibles & $-10 \%$ & $9 \%$ \\
\hline Promociones en planes disponibles & $-11 \%$ & $10 \%$ \\
\hline Oferta de equipos móviles disponibles & $-5 \%$ & $7 \%$ \\
\hline Medios de comunicación de planes y promociones & $-13 \%$ & $6 \%$ \\
\hline Publicidad en internet & $-5 \%$ & $5 \%$ \\
\hline Publicidad radio & $-4 \%$ & $1 \%$ \\
\hline Publicidad en televisión & $-4 \%$ & $3 \%$ \\
\hline Publicidad en redes sociales & $-7 \%$ & $6 \%$ \\
\hline
\end{tabular}

Fuente: elaboración propia.

En contrapeso de los aspectos que deterioran el nivel de recomendación de los usuarios frente a su operador de internet móvil, se identifican dos que contribuyen en gran medida a mejorar este indicador:

- Cumplimiento de la promesa de venta: es un aspecto que afecta de gran forma el NPS si no se cumple, pero también impacta de forma significativamente positiva si el usuario percibe que el operador ha cumplido todo lo que le prometió en el momento de contratar el servicio. Este parece ser el aspecto que más debe ser trabajado por parte de los operadores si quieren mejorar su indicador de NPS en un $+22 \%$.

- Desempeño del servicio para la visualización de videos sin conexión a wifi: los grandes avances en la tecnología de los equipos móviles y en las velocidades ofertadas en el mercado han posicionado la posibilidad de visualizar videos en línea como uno de los requisitos mínimos que los usuarios esperan de su servicio de internet móvil contratado. Si es bien percibido por parte de este, puede generar un impacto del $+15 \%$ en el NPS. 


\section{CONCLUSIONES}

Se establecieron las áreas funcionales que comprenden los aspectos y momentos del ciclo de prestación del servicio de internet móvil más relevantes para monitorear la satisfacción de los usuarios: comercial, canales, producto, precio, upgrade, cancelación y promoción. Estas sirvieron para generar los ítems exploratorios que hacen parte del instrumento de investigación desarrollado.

El instrumento desarrollado fue validado en cuanto a su contenido y pertinencia por un panel de siete expertos, y en cuanto a su lenguaje y claridad por un panel de treinta usuarios del servicio de internet móvil. Estas validaciones respaldan que el instrumento desarrollado permite recolectar información útil para medir y analizar la satisfacción de los usuarios frente al desempeño del servicio prestado por su operador actual.

Resultó factible realizar la aplicación del instrumento a la muestra de usuarios de internet móvil mediante la estrategia de paneles auditados online. Este tipo de trabajo de campo permite recolectar la información de forma más rápida y focalizada, lo que puede ser favorable si se establece efectuar mediciones de corte longitudinal para hacer un monitoreo del desempeño de los operadores a lo largo del tiempo.

El plan de procesamiento y análisis propuesto permite desplegar el análisis de la satisfacción en torno a tres puntos clave: funnel, top of mind y equity. El funnel análisis, que permite conocer el desempeño general del operador en el mercado y que podría complementarse con estudios de top of mind share de mercado e inversión de mercadeo para profundizar en equity de marca; el indicador NPS, que permite medir la satisfacción por medio del nivel de recomendación del usuario respecto a su operador; y el análisis de relación entre el NPS y el desempeño del operador en los diferentes aspectos y momentos del ciclo de prestación del servicio. Este último cobra gran relevancia, ya que permite identificar los focos de acción para trabajar por parte de cada operador para mejorar la satisfacción de sus usuarios.

La propuesta de modelo metodológico para la medición de la satisfacción de los usuarios de internet móvil en Colombia permite ir más allá de los indicadores solicitados por los entes de control, analizando el desempeño de los operadores en todos los aspectos y momentos del ciclo de prestación del servicio y generando el insumo necesario para la puesta en marcha de planes de acción en diversos frentes.

Para dar continuidad a la investigación podrían llevarse a cabo mediciones periódicas que permitan monitorear la satisfacción de los usuarios a lo largo del tiempo y determinar si las acciones llevadas a cabo por los operadores tienen un impacto sobre aquella y cuánto tarda dicho impacto en reflejarse.

Futuros trabajos también podrían centrarse en la revisión del instrumento frente a posibles cambios normativos, de contexto o de mercado que puedan haber surgido y su aplicación para una próxima medición sobre una nueva muestra representativa a nivel nacional calculada a partir del universo de usuarios y penetración del servicio actual.

\section{REFERENCIAS}

Babbie, E., Wagner, W. \& Zaino, J. (2019). Adventures in social research. Data analysis using IBM SPSS Statistics. Los Ángeles, Estados Unidos: SAGE.

Chan, L., Hui, Y., Lo, H., Tse, O., Tso, G. \& Wu, M. (2003). Consumer satisfaction index: New 
practice and findings. European Journal of Marketing, 37(5), 872-909. Recuperado de https://www. semanticscholar.org/paper/Consumer-satisfactionindex\%3A-new-practice-and-Chan-Hui/ade4eb006f6530c31133026ebc168ec5d721ee6f

Comisión de Regulación de Comunicaciones, CRC. (2011). Resolución No. 3066 de 2011. Recuperado de https://www.crcom.gov.co/resoluciones/00003066. pdf

Comisión de Regulación de Comunicaciones, CRC. (2016). Resolución No. 5050 de 2016. Recuperado de https://www.crcom.gov.co/es/pagina/resolucion-crc5050-de-2016

Comisión de Regulación de Comunicaciones, CRC. (2017). Resolución No. 5111 de 2017. Recuperado de https://www.crcom.gov.co/resoluciones/00005111. pdf

Comisión de Regulación de Comunicaciones, CRC. (2018). Medición de la calidad de los servicios desde la experiencia de los usuarios 2017. Recuperado de https://www.crcom.gov.co/es/pagina/medicioncalidad-2017

Comisión de Regulación de Comunicaciones, CRC. (2017). Reporte de industria sector TIC 2017. Recuperado de https://www.crcom.gov.co/recursos_user/ reporteindustria2017.pdf

Deloitte. (2018). Consumo móvil en Colombia. Siempre conectados: ¿Bendición o maldición? Recuperado de https://www2.deloitte.com/content/dam/Deloitte/ co/Documents/technology-media-telecommunications/Consumo\%20movil\%202018.pdf
Eklöf, J. (2000). European Customer Satisfaction Index. Pan-European telecommunication sector report based on the pilot studies 1999. Estocolmo, Suecia: European Organization for Quality and European Foundation for Quality Management.

Fornell, C. (1992). A National Customer Satisfaction Barometer: The Swedish experience. Journal of Marketing, 56(1), 6-21. DOI: https://doi. org/10.1177/002224299205600103

Fornell, C., Johnson, M., Anderson, E. \& Bryant, B. (1996). The American Customer Satisfaction Index: Nature, purpose, and findings. Journal of Marketing, 60(4), 7-18. DOI: https://doi. org/10.1177/002224299606000403

Hernández-Sampieri, R., Fernández, C. \& Baptista, P. (2014). Metodología de la investigación. México D.F., México: McGraw-Hill.

Ministerio de Transportes y Telecomunicaciones de Chile (2019). Estudio de Satisfacción de Usuarios de Servicios de Telecomunicaciones. Recuperado de https://www.subtel.gob.cl/wp-content/ uploads/2019/01/estudio_sat_diciembre_2018.pdf

Unión Internacional de Telecomunicaciones - UIT. (2018). Measuring the information society report. Volumen 1. Recuperado de https://www.itu.int/en/ITUD/Statistics/Documents/publications/misr2018/ MISR-2018-Vol-1-E.pdf

Johnson, M., Gustafsson, A., Andreassen, T., Lervik, L. $\&$ Cha, J. (2001). The evolution and future of national customer satisfaction index models. Journal of Economic Psychology, 22(2), 217-245. DOI: https:// doi.org/10.1016/S0167-4870(01)00030-7 
Kotler, P. (2001). Dirección de mercadotecnia: análisis, planeación, implementación y control. Evanston, Estados Unidos: Pearson.

Laitinen, M. (2018). Net Promoter Score as Indicator of Library Customer's perception. Journal of Library Administration, 58(4), 394-406. DOI: https://doi.org /10.1080/01930826.2018.1448655

Ministerio de Tecnologías de la Información y las Comunicaciones - MinTIC. (2018). Boletín trimestral de las TIC. Cifras segundo trimestre de 2018. Recuperado de https://colombiatic.mintic.gov.co/679/ articles-80413_archivo_pdf.pdf

Ospina, S. y Gil, I. (2011). Índices nacionales de satisfacción del consumidor. Una propuesta de revisión de la literatura. Cuadernos de Administración, 24(43), 35-57. Recuperado de http://www.scielo.org.co/pdf/ $\mathrm{cadm} / \mathrm{v} 24 \mathrm{n} 43 / \mathrm{v} 24 \mathrm{n} 43 \mathrm{a} 03 . \mathrm{pdf}$

Reichheld, F. (2003). The One Number You Need to Grow. Harvard Business Review. Recuperado de https://hbr.org/2003/12/the-one-number-you-needto-grow

Velandia, F., Ardón, N. \& Jara, M. (2007). Satisfacción y calidad: análisis de la equivalencia o no de los términos. Revista Gerencia y Politicas de Salud, 6(13), 139-168. Recuperado de https://revistas.javeriana. edu.co/index.php/gerepolsal/article/view/2695 